Technical Note

\title{
Exact Solution of the Friction Circle Method
}

\author{
Jozsef Garai ${ }^{1}$
}

\begin{abstract}
The modified friction circle method is still an important tool in slope-stability investigations. The theoretical soundness of this method was investigated, and it is shown here that there is theoretical inconsistency and that the radius of the friction circle is incorrect. Using fundamental relationships of classical mechanics, the exact solution is derived from first principles. DOI: 10.1061/(ASCE) GM.1943-5622.0000618. ( $) 2015$ American Society of Civil Engineers.
\end{abstract}

Author keywords: Friction circle; Modified friction circle; Friction circle method; Slope stability; Slope-stability investigation.

\section{Introduction}

The application of the friction circle method to geotechnical problems was proposed by Glennon Gilboy and Arthur Casagrande (Taylor 1937). It is assumed that the Mohr-Coulomb failure criterion is valid and that the failure surface is cylindrical. Equilibrium conditions are applied to a rigid body with a unit width, failing along a circular surface. The principle of the method is that the intergranular forces are in an obliquity of $\varphi$ to the circular surface at failure, where $\varphi$ is the angle of the internal friction of the soil. When the length of the arc is divided into small elements, the line of action of the intergranular forces acting on these elements can be defined by a tangent to the friction circle drawn around the center of the sliding circle (Fig. 1). The radius of the friction circle is

$$
R_{f}=R \sin \varphi
$$

where $R$ is the radius of the sliding circle. The friction circle is a graphical tool that defines the line of action of the intergranular forces at any given point on the sliding circle in a convenient way. In geotechnics, the method is used primarily for slope-stability investigations in homogeneous soil when both cohesive and frictional components have to be considered in the calculations (e.g., Abramson et al. 2002; Das 2006).

The pitfall of the original method is that the resultant of intergranular forces falls outside of the friction circle (Fig. 2). Thus, the radius of the friction circle used for the resultant should be bigger than $R_{f}=R \sin \varphi$. Taylor (1937) introduced a constant multiplier $[K]$ to compensate for this difference. The multiplier is the function of the central angle $[\alpha]$ and the stress distribution, which is usually assumed to be sinusoidal (Taylor 1937; Murthy 2002). This semi-empirical approach is known as the modified friction circle method. Using this method, Taylor developed charts for slope stability (Taylor 1937). Despite the overwhelming success of computer methods in current geotechnics, these charts, with some modifications, are still used frequently in practice (e.g.,

\footnotetext{
${ }^{1}$ Associate Professor, Dept. of Civil Engineering, Univ. of Debrecen Ótemetö u. 2-4, 4028 Debrecen, Hungary. E mail: garai.jozsef@eng .unideb.hu

Note. This manuscript was submitted on January 12, 2015; approved on October 6, 2015; published online on riod open until $\square \square \square \square$; separate discussions must be submitted for individual papers. This technical note is part of the International Journal of Geomechanics, (C) ASCE, ISSN 1532-3641.
}

Michalowski 2002; Steward et al. 2011). Thus, the friction circle method is still an important tool in slope-stability investigations. In this study, the theoretical soundness of the friction circle method is investigated.

\section{Modified Friction Circle Method}

The forces acting on the failing soil slope body are

- The total weight $[W]$ calculated from the mass above the sliding/ failure circle;

- The resultant of the intergranular forces $[P]$; and

- The resultant of the cohesive forces $[C]$.

Other possible forces, such as neutral, seepage, and seismic, have no significance on the outcome of this investigation; therefore, for simplicity, these forces are not considered here.

The actuating force is the total weight, which acts through the mass center. The resistive forces are the cohesive and the intergranular. The resultant of the mobilized cohesive forces $\left[C_{m}\right]$ is the vector sum of the mobilized cohesive forces that act along the sliding circle (Fig. 3). The magnitude of this force is calculated as

$$
C_{m}=c_{m} L_{\text {chord }}, \text { where } c_{m}=\frac{c}{F_{s}}
$$

where $c$ represents the effective unit cohesion of the soil; $c_{m}$ is the effective mobilized unit cohesion of the soil; $L_{\text {chord }}$ is the length of the chord of the sliding circle; and $F_{s}$ is the factor of safety. From the vector sum of the cohesive forces $\left[C_{m i}\right]$ acting along the arc, it can be seen that the direction of the resultant of the cohesive force is parallel with the direction of the chord. The line of action is distanced $\left[R_{c}\right]$ from the center of the circle. This distance is defined from momentum equilibrium of the cohesive forces to the center of the trial circle and is calculated as

$$
R_{c}=\frac{L_{\text {arc }}}{L_{\text {chord }}} R
$$

where $L_{\text {arc }}$ is the length of the arc of the sliding circle. The frictional component of the resistive forces acts against the movement along the failure surface. The line of action of the resultant of the intergranular forces is defined by the tangent line to the modified friction circle. When the force that represents the total weight and the directions of the resultants of the intergranular and the cohesive forces are known, the magnitude of these forces can be determined from equilibrium conditions (Fig. 3). 


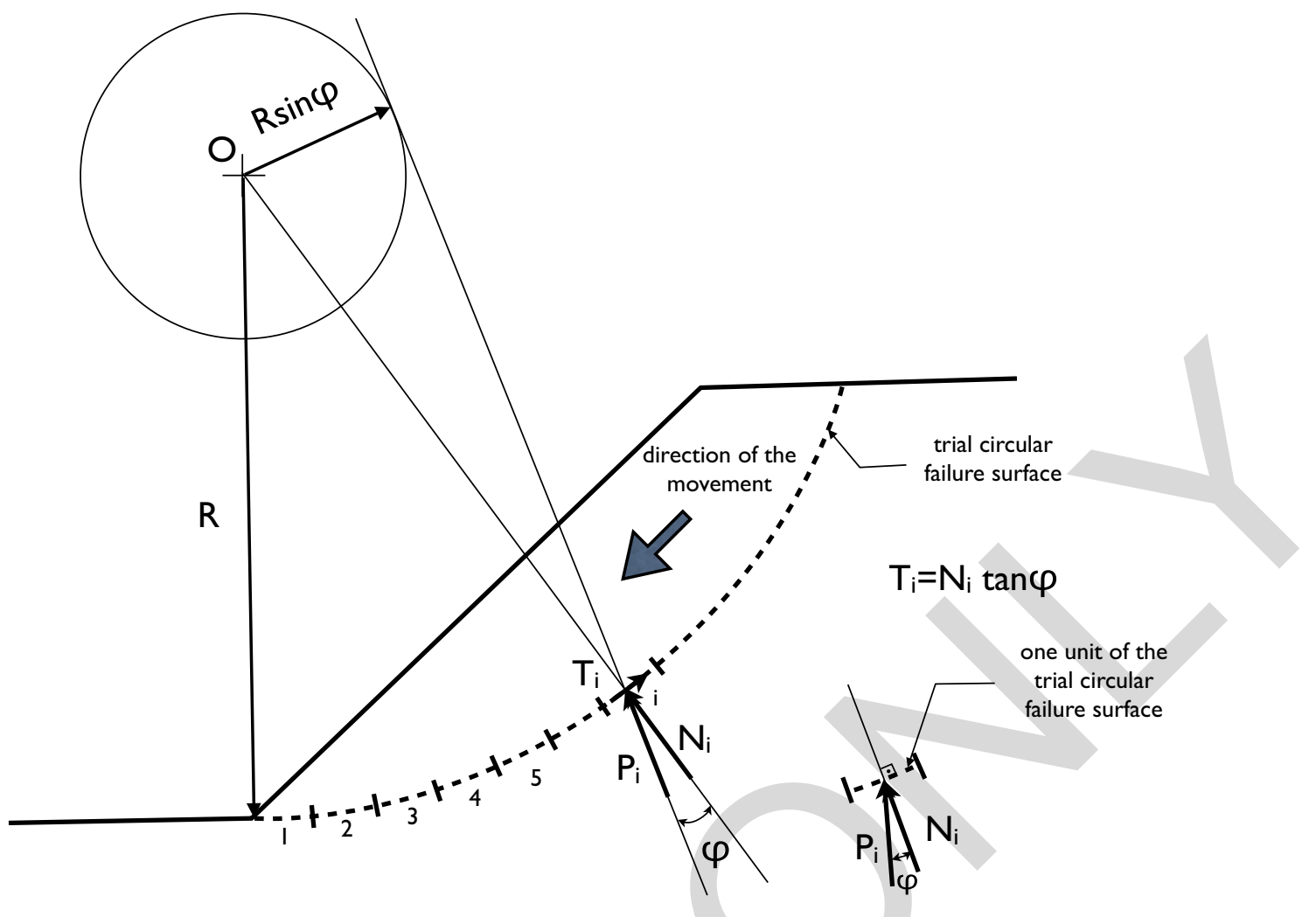

Fig. 1. Principles of the friction circle method

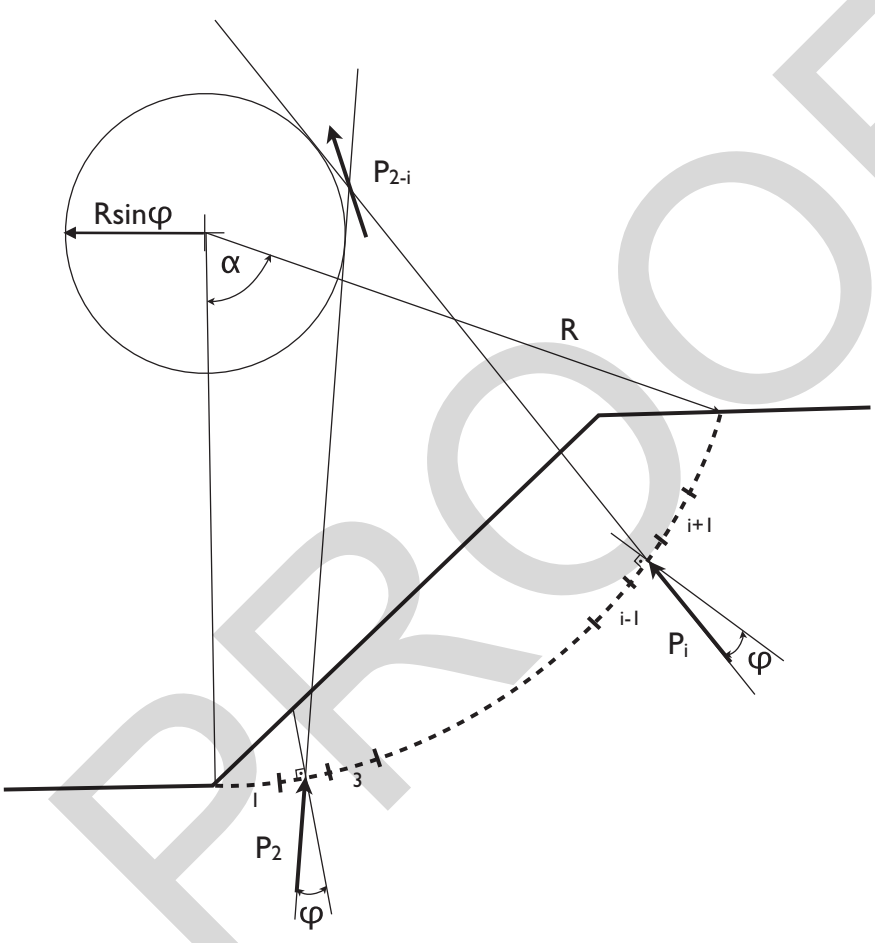

F2:1 Fig. 2. Resultant of the intergranular forces tangent to a circle bigger $\mathrm{F} 2: 2$ than the friction circle
The factor of safety can be defined by assuming that (1) the frictional strength is completely mobilized, (2) the friction and cohesion strength are mobilized partially or equally, or (3) by using the ratio of the residual and actuating momentums.
If the frictional strength has been fully mobilized (Assumption 1), then the factor of safety can be defined as

$$
F_{s}=\frac{c}{c_{m}}
$$

The factor of safety with respect to the two resisting components of the strength, cohesion and friction, can be defined separately (Assumption 2) as the ratio between the residual and mobilized parts of the strength as

$$
F_{s}(c)=\frac{c}{c_{m}} \text { and } F_{s}(\varphi)=\frac{\operatorname{tg} \varphi}{\operatorname{tg} \varphi_{m}}
$$

Assuming the same mobilization for the friction and cohesion strength gives the factor of safety as

$$
F_{s}(c)=F_{s}(\varphi)
$$

The ratio of the total resisting and activating momentum (Assumption 3) can also be used to define the factor of safety (Frohlich 1954) as

$$
F_{s}=\frac{M_{r}}{M_{a}}
$$

where $M_{r}$ represents the total resisting moments of the available shear strength about the center of the trial circle; and $M_{a}$ is the total activating moment around the same center.

The minimum value of the factor of safety is defined by a hitand-miss method by calculating the factor of safety for the number of slip surfaces. The lowest factor of safety can be found by drawing contour lines, and the minimum value defines the most likely surface of failure. 


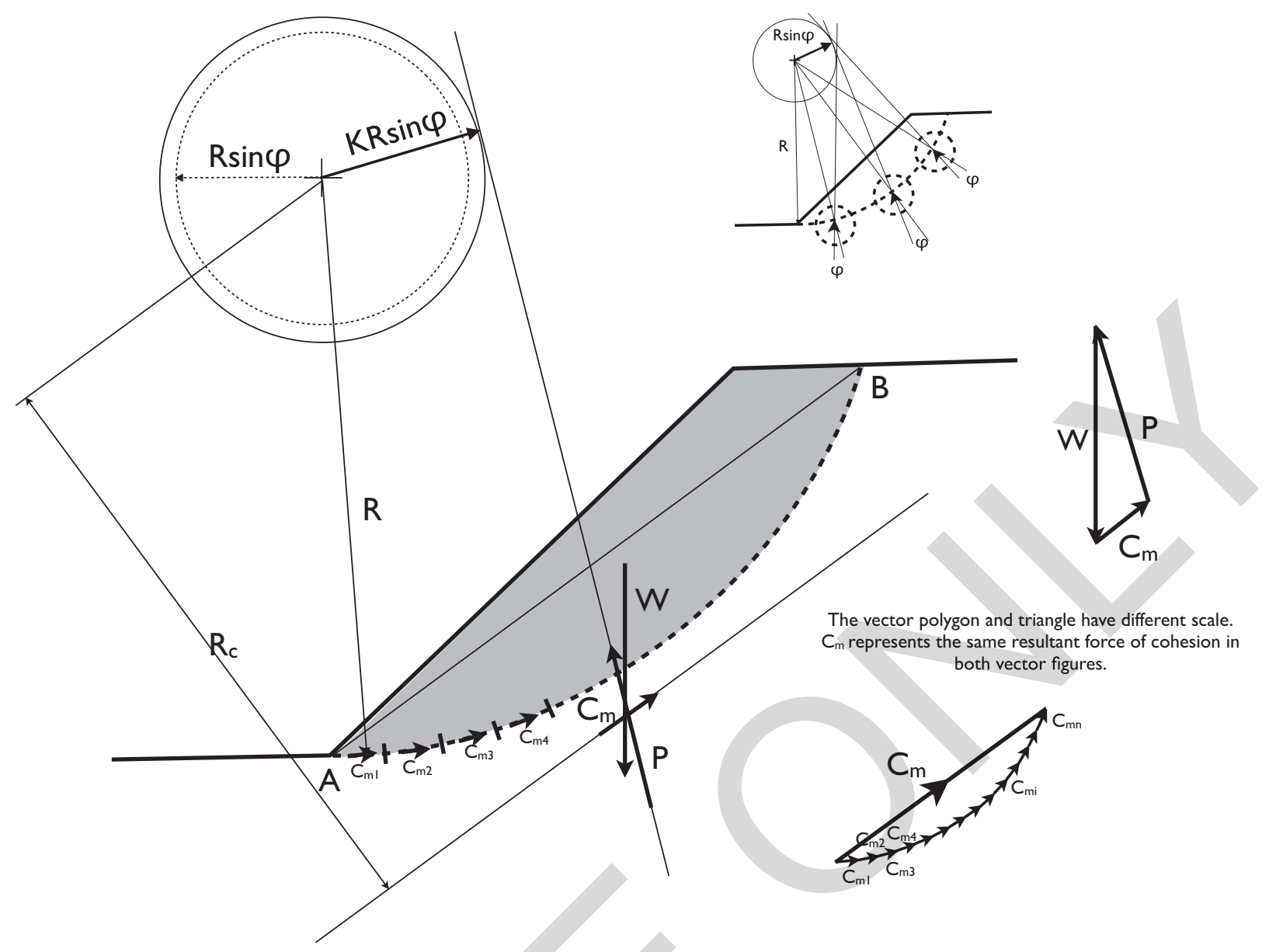

\section{Theoretical Problems with the Method} sound theory of mechanics. here.
In this simplified case, stated earlier, only three forces are acting on this sliding body. Among these forces, the magnitude and the line of action of the weight are known from the geometry of the sliding slope and the unit weight of the soil. In the assumed homogeneous soil, the resistance of the cohesion forces along the trial circle is uniform. The vector sum of the cohesive forces gives the magnitude and the direction of the line of action of the resultant cohesive force. The distance of the line of action of the resultant of the cohesive forces can be defined from moment equilibrium to the center of the trial circle as described earlier [Eq. (3)]. By using the fundamental relationships of mechanics the magnitude, the direction and the line of action of the resultant of the cohesive forces can be determined. It is concluded that the calculations of the resultants of the weight and the cohesive forces are based on a

The equilibrium of the system requires that the resultant of the intergranular forces satisfy two conditions. First, the forces must be concurrent; therefore, the resultant of the intergranular forces must intersect the cohesion and the weight force vectors at their intersection. Also, in accordance with the friction circle method, the direction of the resultant of the intergranular forces should be the tangent line to the modified friction circle. The first condition is deduced from the fundamental equilibrium requirement. The theoretical justification of the second requirement is investigated
The value of $K$ is the function of the central angle $[\alpha]$ and the stress distribution (Taylor 1937). The simplest case is the uniform stress distribution, which is investigated here. The normal forces acting on the surface of the sliding circle are symmetrical to the line of symmetry of the arc (Fig. 4). In the case of uniform stress distribution along the failing circle, the resultant of the normal forces acts on the line of symmetry, or more specifically, the resultant of the normal forces is perpendicular to the cord of the sliding circle, and the line of action intersects the cord in the middle. The uniform normal forces induce uniform frictional forces along the sliding surface of the arc. The resultant of these friction forces can be determined in the same way as the resultant of the cohesion forces. The direction of the line of action of the resultant of the frictional forces is parallel with the chord, and the line of action coincides with the line of action of the resultant of the cohesion forces (Fig. 4).

To be in equilibrium, the three forces (weight, cohesion, and intergranular) must be concurrent and intersect each other at the same point. Thus, the line of action of the intergranular force must go through the intersection of the weight and cohesion forces. The angle between the resultant of the intergranular forces and the resultant of the normal forces $[\beta]$ varies depending on the location of this intersection. The angle between these forces represents the internal friction of the soil, which should not be effected by the position of the intersection of the resultants of the weight and cohesion. Thus, this angle must be defined uniquely. On the basis of this contradiction, it is concluded that the modified friction circle method is theoretically inconsistent. 


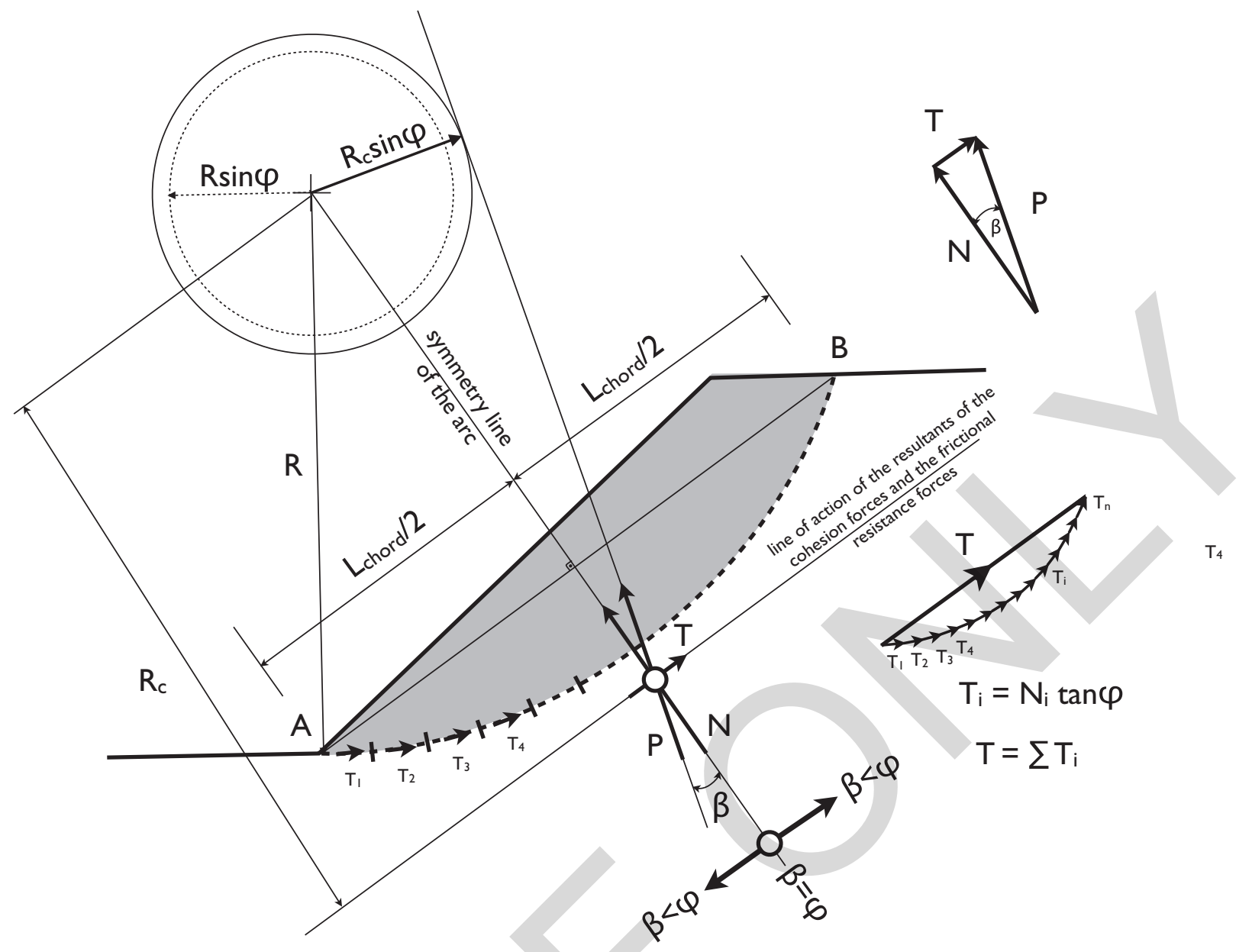

Fig. 4. The currently used method is inconsistent because the angle between the resultant of the normal forces and intergranular forces is not unique

\section{Exact Radius of the Friction Circle}

All the normal forces that act along the sliding circle are concurrent, and the lines of action of the forces go through the center of the sliding circle. The line of action of the resultant of these forces, therefore, must intersect the same point. Thus, the line of action of the resultant of the normal forces goes through the center of the sliding circle. The line of action of the intergranular forces intercepts the cohesion and the weight forces at the same point. Thus, the line of action of the resultant of the normal forces must also intercept this point.

The normal force $[N]$ and the induced frictional shear resistance [T] form an orthogonal system because $T$ is always perpendicular to $N$, and vice versa. By rotating this orthogonal system and projecting the original components onto the new system gives the new components as

$$
T^{\prime}=T \cos \delta \text { and } N^{\prime}=N \cos \delta
$$

where $\delta$ is the angle of rotation. Please notice that the ratio of the projected new components is the same as the ratio in the original system, because dividing the two equations [Eq. (8)] gives

$$
\frac{T^{\prime}}{N^{\prime}}=\frac{T}{N}
$$

The angle of rotation $\delta$ can have any value without violating this outcome. Because all the intergranular forces are in an obliquity of $\varphi$ to the circular surface at failure and the ratio between the frictional shear resistance force [Ti] and the corresponding normal force [Ni] is equal to $\tan \varphi$, the ratio of the resultants of $T$ and $N$ should remain the same regardless of the original directions of the components. Thus, the angle between the resultants of $N$ and $P$ must be the same as $\varphi$.

Because all of the intergranular normal forces $\left[P_{i}\right]$ are in an obliquity of $\varphi$ to the sliding plain, the resultant of the intergranular forces and the resultant of the normal forces must intersect at an angle of $\varphi$. This condition can be satisfied and determined graphically by a friction circle, which has the radius of

$$
R_{f-c}=R_{i} \sin \varphi,
$$

where $R_{i}$ is the distance between the center of the sliding circle and the interception of the line of action of the resultants of the cohesion and the weight (Fig. 5). The defined resultant of the intergranular forces is consistent with the initial assumptions and the fundamental equations of mechanics.

The error that results from the incorrect radius of the friction circle (the currently used method) results in a difference of a few percent in the factor of safety depending on the geometry of the slope, the parameters of the soil $(\gamma, \varphi, c)$, and the definition of the factor of safety. The introduced error always reduces the value of the factor of safety. Thus, the currently used method underestimates the factor of safety in comparison to the theoretically correct solution. 


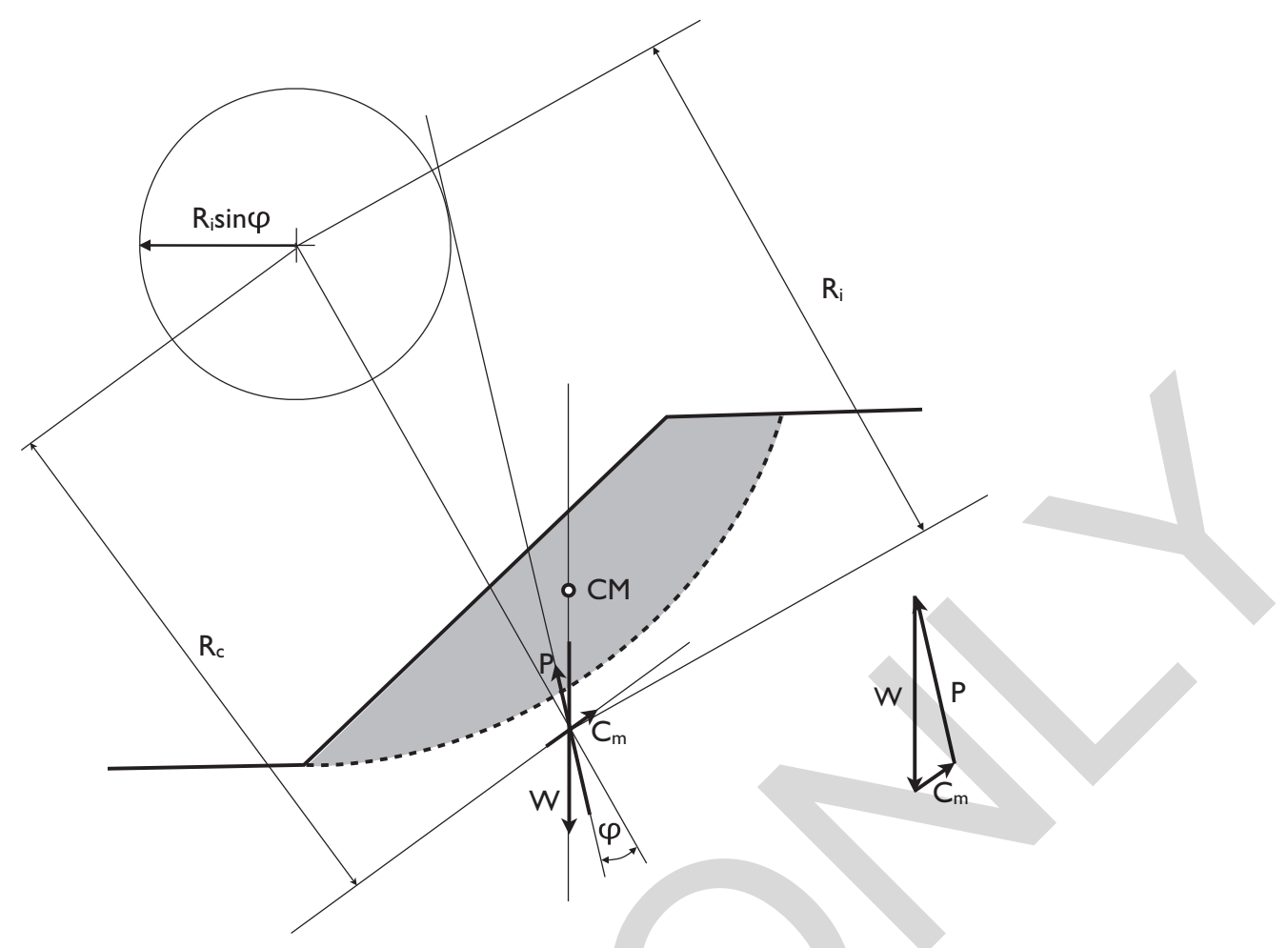

\section{1}

192

\section{Conclusions}

It has been shown that the currently used modified friction circle method is inconsistent with theory. The conditions deduced from fundamental equations of mechanics, which must be satisfied for equilibrium, are as follows:

- The line of action of the resultant of the normal forces must intersect the center of the sliding circle;

- The line of action of the resultant of the frictional shear resistance forces, for uniform normal force distribution, is identical with the line of action of the resultant of the cohesive forces;

- Projecting the two orthogonal force components, acting along the sliding circle, into a new orthogonal system of the resultants of the normal and frictional shear resistance forces leaves the ratio between the components unchanged; thus, the ratio between frictional shear resistance and normal forces is uniquely defined by the angle of the internal friction of the soil; and

- The equilibrium of the sliding slope requires the resultants of the weight, the cohesion, and the intergranular forces to be concurrent.

It is shown that these conditions can be satisfied by using a friction circle with a radius of $R_{f-c}=R_{i} \sin \varphi$, where $R_{i}$ is the distance between the center of the sliding circle and the interception of the line of action of the resultants of the cohesion and the weight forces.

The friction circle method with the derived new friction circle radius is consistent and complies with all of the fundamental equations of mechanics and gives an exact solution for two-dimensional slope-stability investigations.

\section{Notation}

The following symbols are used in this paper:

$C=$ resultant force from cohesion (in $\mathrm{N}$ );

$c=$ effective cohesion of the soil (in $\left.\mathrm{N} / \mathrm{m}^{2}\right)$;
$C_{m}=$ resultant force from mobilized cohesion (in $\mathrm{N}$ );

$c_{m}=$ effective mobilized cohesion (in $\mathrm{N} / \mathrm{m}^{2}$ );

$F_{s}=$ factor of safety (dimensionless);

$K=$ constant multiplier for the modified friction circle radius (dimensionless);

$L_{\text {arc }}=$ length of the arc of the sliding circle (in m);

$L_{\text {cord }}=$ length of the chord of the sliding circle (in m);

$M_{a}=$ total activating moment around the center of the sliding circle (in Nm);

$M_{r}=$ total resisting moments of the available shear strength about the center of the sliding circle (in $\mathrm{Nm}$ );

$N=$ normal force (in $\mathrm{N})$;

$P=$ intergranular force (in $\mathrm{N}$ );

$R=$ radius of the sliding circle (in $\mathrm{m}$ );

$R_{c}=$ perpendicular distance between the center of the sliding circle and the line of action of the resultant of the cohesion forces (in $\mathrm{m}$ );

$R_{c-i}=$ distance between the center of the sliding circle and the interception of the resultants of the weight and cohesion forces (in $\mathrm{m}$ );

$R_{f}=$ radius of the friction circle calculated by Eq. (1) (in m);

$R_{f-c}=$ radius of the derived theoretically correct friction circle (in $\mathrm{m}$ );

$R_{f-m}=$ radius of the modified friction circle (in $\mathrm{m}$ );

$T=$ tangential force/resistant force from the internal friction (in N);

$W=$ total weight of the soil above the sliding circle (in $\mathrm{N}$ );

$\alpha=$ central angle of the sliding slope (in degrees);

$\beta=$ angle between the resultant normal forces and the resultant intergranular force (in degrees);

$\delta=$ angle of the rotation of the orthogonal system (in degrees); 


$$
=
$$

